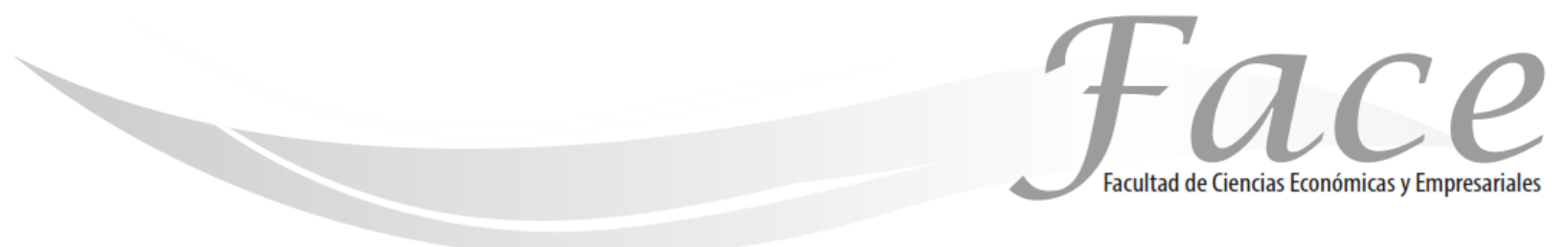

ISSN Impreso: 1794-9920

ISSN Electrónico: 2500-9338

Volumen $19-\mathrm{N}^{\circ} 2$

Año 2019

Págs. $24-40$

\title{
LA INVESTIGACIÓN CIENTÍFICA, LOS CUERPOS ACADÉMICOS Y LAS REDES DE INVESTIGACIÓN
}

\author{
Indira Loyda Cordero Dámaso * \\ ORCID: https://orcid.org/0000-0003-3972-1262 \\ Ramón Almazo Domínguez ** \\ ORCID: https://orcid.org/0000-0002-7249-6143 \\ Sonia González Bravo *** \\ ORCID: https://orcid.org/0000-0002-7734-5088 \\ Rosario Ortiz Cabrera **** \\ ORCID: https://orcid.org/0000-0001-6466-017X
}

Fecha de Recepción: Julio 27 de 2019

Fecha de Aprobación: Septiembre 28 de 2019

\section{Resumen:}

La planta docente con grado de Maestría y Doctorado, contratados con la categoría de asignatura o medio tiempo, por tiempo determinado o definitivo, en todas las Instituciones del Nivel Superior, sean Públicas o Privadas, por carecer del perfil deseable reconocido Institucionalmente, únicamente son considerados como colaboradores. Si las Instituciones Educativas del Nivel Superior, modifican sus criterios, relacionados con el perfil deseable, respecto a los miembros investigadores de los Cuerpos Académicos, Redes y Líneas de Investigación Científica, ¿Los Docentes con grado de Maestría o Doctorado, por Asignatura 0 Medio Tiempo, contratados por tiempo determinado o definitivo, en la Benemérita Universidad Autónoma de Puebla y en general del nivel superior sean Públicas o Privadas, estarían en posibilidad de integrarse como miembros investigadores de los Cuerpos Académicos (CA), Redes (RI) y Líneas de Investigación científica?. Se trata de una investigación. El presente trabajo, tiene como objetivo realizar un análisis del por qué no se incluye a la totalidad de los docentes, actualmente guía, coordinador, orientador, facilitador e instructor del proceso aprendizaje-enseñanza, y sobre todo, de las limitantes que imperan en la integración como miembros investigadores de los "Cuerpos académicos (CA), Redes (RI) y Líneas de Investigación Científica", que por carecer del "perfil deseable", beneficio exclusivo de los docentes con categoría de Tiempo Completo, con grado académico de Doctorado, Maestría y con tipo de contratación como Definitivos en la BUAP.

Palabras clave: cuerpos académicos, grupos de investigación, redes científicas, docente-investigador, investigación.

* Docente-investigador, Complejo Regional Mixteca1, Complejo Regional Centro, Benemérita Universidad Autónoma De Puebla. Contacto: indi0482@hotmail.com.

Docente-investigador, Complejo Regional Mixteca1, Complejo Regional Centro, Benemérita Universidad Autónoma De Puebla. Contacto: ramon.almazo@correo.buap.mx.

Docente-investigador, Complejo Regional Mixteca1, Complejo Regional Centro, Benemérita Universidad Autónoma De Puebla. Contacto: gobscp47@gmail.com.

Docente-investigador, Complejo Regional Mixteca1, Complejo Regional Centro2 Benemérita Universidad Autónoma De Puebla. Contacto: rosario.ortiz@correo.buap.mx. 


\title{
SCIENTIFIC RESEARCH, ACADEMIC GROUPS AND RESEARCH NETWORKS
}

\begin{abstract}
:
The teaching staff with a Master's and Doctorate degree, contracted with the category of subject or part-time, for a definite or definitive time, in all Institutions of the Higher Level, whether Public or Private, for lacking the desirable profile recognized Institutionally, are only considered as collaborators If the Higher Level Educational Institutions modify their criteria, related to the desirable profile, with respect to the research members of the Academic Bodies, Networks and Lines of Scientific Research, Do Teachers with a Master's or Doctorate degree, by Subject or Half Time, hired for a definite or definitive time, at the Benemérita Universidad Autónoma de Puebla and in general of the higher level, whether Public or Private, would be able to integrate as research members of the Academic Bodies (CA), Networks (RI) and Research Lines scientific ?. It is an investigation. The purpose of this work is to carry out an analysis of why all teachers are not included, currently a guide, coordinator, counselor, facilitator and instructor of the learning-teaching process, and above all, of the limitations that prevail in the integration as research members of the "Academic bodies (CA), Networks (RI) and Lines of Scientific Research", which for lacking the "desirable profile", exclusive benefit of teachers with a Full Time category, with an academic degree of Doctorate, Master's degree and with type of hiring as Definitive in the BUAP.
\end{abstract}

Keywords: Academic Groups, Research Groups, Scientific Networks, Teacher-Researcher, Research

\section{PESQUISA CIENTÍFICA, GRUPOS ACADÊMICOS E REDES DE PESQUISA}

\section{Resumo:}

Os docentes com mestrado e doutorado, contratados com a categoria de disciplina ou em regime de meio período, por tempo determinado ou definitivo, em todas as Instituições de Nível Superior, sejam elas Públicas ou Privadas, por falta do perfil desejável reconhecido Institucionalmente, são somente considerados colaboradores Se as Instituições de Ensino Superior modificarem seus critérios, relacionados ao perfil desejável, em relação aos membros da pesquisa dos Órgãos Acadêmicos, Redes e Linhas de Pesquisa Científica, Professores com Mestrado ou Doutorado, por Assunto ou A Half Time, contratada por tempo determinado ou definitivo, na Benemérita Universidad Autónoma de Puebla e, em geral, de nível superior, público ou privado, poderia integrar-se como membros de pesquisa dos Órgãos Acadêmicos (CA), Redes (RI). ) e Linhas de Pesquisa científicas? É uma investigação. $O$ objetivo deste trabalho é realizar uma análise de por que todos os professores não estão incluídos, atualmente um guia, coordenador, conselheiro, facilitador e instrutor do processo de ensino-aprendizagem e, acima de tudo, das limitações que prevalecem na integração como membros de pesquisa dos "Órgãos Acadêmicos (CA), Redes (RI) e Linhas de Pesquisa Científica", que por falta do "perfil desejável", benefício exclusivo de professores com categoria de tempo integral, com grau acadêmico de doutorado, mestrado e com o tipo de contratação como Definitivo no BUAP.

Palavras-chave: Grupos Acadêmicos, Grupos de Pesquisa, Redes Cientificas, Professor-Pesquisador, Pesquisa 


\section{INTRODUCCIÓN:}

A partir de la década de los noventa, el Subsistema de Educación Superior Mexicano se ha venido transformando sustancialmente debido a la implementación de un paquete de políticas públicas para el subsector, derivadas del examen que en esta materia realizó la Organización para la Cooperación y el Desarrollo Económico (OCDE) en nuestro país durante los años comprendidos de 1994-1996, destacando el desarrollo del personal y el de la calidad, que dieron lugar, respectivamente al Programa de Mejoramiento del Profesorado (PROMEP, actualmente PRODEP) y la Agenda Mexicana para la Mejora de la Calidad de la Educación Superior. Posteriormente, se diseñaron una serie de acciones planeadas para el periodo de 1996-2006, en el caso específico del Programa de Mejoramiento del Profesorado, teniendo como eje central: la constitución y consolidación de Cuerpos Académicos, Redes y Líneas de Investigación Científica, en las Direcciones de Educación Superior (DES). Estas acciones se articularon a otros rubros a partir del año 2000, dando lugar a ejercicios de planeación institucional que se concretaron en su momento a los Programas Integrales de Fortalecimiento Institucional.

En cada una de las Instituciones y Direcciones de Educación Superior participantes se lograron avances en tales rubros "-aun cuando no se han evaluado- se pueden apreciar como significativos, la actual administración federal ha decidido su permanencia como parte de la estrategia educativa para que se cumplan los objetivos de eficiencia y calidad en nuestro país, considerando conveniente hacer un corte al término de su primera etapa para revisar su pertinencia, relevancia e impacto, particularmente lo relacionado con las propuestas de transitar al nivel de consolidación que se pretende logren los cuerpos académicos, y de ahí a la formación de redes de colaboración" 1.

\section{Panorama General:}

Actualmente, con el sistema económico imperante como lo es la globalización, producto del neoliberalismo, cuya consecuencia son las exigencias de los Organismos e Instituciones de carácter Internacional, como el Banco Mundial (BM), la Organización para la Cooperación y el Desarrollo Económico (OCDE), la Organización de las Naciones Unidas para la Educación, la Ciencia y la Cultura (UNESCO), entre otros, que demandan que las Instituciones Educativas del Nivel Superior, sean Públicas o Privadas, mayor preparación y participación de sus docentes, actualmente guía, coordinador, orientador, facilitador e instructor del proceso aprendizaje-enseñanza, independientemente de su grado de preparación académica, categoría y tipo de contratación, que no reúnen el perfil deseable, se debe considerar su integración como miembros investigadores de los Cuerpos Académicos (CA), Redes de Investigación (RI) y Líneas de Investigación Científica, quienes además adquieren la obligación ineludible de proporcionar a sus estudiantes, futuros profesionistas capacitación de calidad y eficiencia, debiendo contar para ello, con programas debidamente certificados, mismo que implica la construcción de modelos académicos que permiten enfrentar y cumplir con los objetivos y retos propuestos, mejorando su funcionamiento, dentro del nuevo orden mundial en el sistema educativo, Institución Educativa, que no puede permanecer ajena a los citados requerimientos.

Ante tales circunstancias, las Instituciones de Educación Superior satisfacen, en la medida de sus posibilidades, dichos requerimientos conforme a su capacidad, fortaleza, calidad, eficacia y eficiencia; aunado a lo anterior, la Asociación Nacional de Universidades e Instituciones de Educación Superior 
Mauricio Ortigosa Hernández

(ANUIES) en coordinación con la Secretaría de Educación Pública Federal, con la finalidad de cumplir con el rubro de investigación científica, diseñaron el Programa para el Desarrollo Profesional Docente, para el tipo Superior (PROMEP, actualmente PRODEP), respecto del cual es determinante la calidad y eficiencia de la investigación científica, que realizan primordialmente, una mínima parte en los profesores de carrera, es decir, los profesores de Tiempo Completo, con perfil deseable, que son reconocidos Institucionalmente, para dar respuesta a las necesidades y propósitos de la investigación científica, sobre todo relacionados con la integración de los miembros investigadores de los "Cuerpos Académicos (CA), Redes (RI) y Líneas de Investigación Científica.

\section{MARCO TEÓRICO. Conceptos:}

Cuerpos Académicos: "Un Cuerpo Académico está integrado por un conjunto de profesores investigadores que comparten una o más líneas de estudio orientados a la generación y aplicación de nuevos conocimientos, en temas disciplinares 0 multidisciplinares y que, además inciden en brindar una educación de calidad a los alumnos"2

Los cuerpos académicos: son grupos de profesores de tiempo completo que comparten una 0 varias líneas de generación o aplicación innovadora del conocimiento (investigación o estudio) en temas disciplinares o multidisciplinares y un conjunto de objetivos y metas académicas. Adicionalmente atienden los Programas Educativos (PE) afines a su especialidad en varios niveles"3

\footnotetext{
${ }^{2}$ Gaceta Universidad. Órgano Oficial de la Benemérita Universidad Autónoma de Puebla. No. 132. 2010, p 16

${ }^{3}$ www.uthh.edu.mx/file manager/doc
}

Los anteriores conceptos, más que definir a los Cuerpos Académicos, indican su forma de integración y sus facultades o competencias que deben ser afines. Conforme al concepto de Red: La red se distingue de otros términos que se han usado históricamente en conjunto con Escuelas y otras Instituciones Educativas, en sus arreglos organizacionales y los modos de comprender la innovación y el cambio. Esta acentúa la idea de "comunidad" como elemento común y el principio de unión entre Instituciones. Hamaláinen, T. and Schienstock, G, citado por la Dra. Blanca Heredia Rubio, afirma que "La investigación en redes muestra que pueden tomar una amplia variedad de formas, y que hay poco consenso sobre una definición apropiada" ... Las redes en general pueden ser diferenciadas por su alcance geográfico y pueden ser locales, regionales, nacionales o internacionales"4.

\section{Redes de investigación científica:}

"Por lo que se refiere al ámbito académico, las redes suelen entenderse como "mecanismo de apoyo, intercambio e información que atraviesan fronteras y brindan un gran dinamismo a partir de la conjunción de intereses respecto a una temática o problema" (Reynaga, 1996). En referencia la red académica, puede concebirse como un mecanismo de apoyo, de intercambio de información y una comunidad de comunicación horizontal, cuya base es una red social, un tejido, una madeja compleja en la que se sinergizan -a través de interacciones entre vínculosdinamismos, intereses, fuerzas, energías y puntos de apoyo y encuentro (nodos), con el propósito principal de dialogar, encontrar respuestas, construir conocimientos y unirse en la búsqueda o creación de soluciones respecto a una temática o problema.

\footnotetext{
${ }^{4}$ Heredia Rubio, Blanca. Innovación en las escuelas. Primera ed. Edit. Colección: Antologías didácticas INITE-OCDE. México, 2008. p 227 y 228.
} 
La importancia de una red, sobre todo de una red académica, es enorme porque permite a los académicos trabajar con flexibilidad, cooperativamente, en el desarrollo académico, científico, técnico, social y cultural en una comunidad, equipo, grupo o región. Permite la integración para la solución de problemas y temáticas comunes, extiende beneficios a funcionarios, educadores, profesores, empresarios, sindicatos; puede constituirse por instituciones, secretarías, centros de investigación; facilita el intercambio de datos, información, conocimiento, y propicia la reflexión. Es un medio para crear fuentes de financiamiento y ofrece una herramienta a la comunidad. Tiene como fines intercambiar, construir, apoyar conocimientos, abonar a la solución de problemas, incrementar el número de investigadores, fortalecer posibilidades.

Y como objetivos propiciar el uso prioritario de la infraestructura disponible para la interconexión de las redes existentes en la región e interconectar redes de información, de bibliotecas, de centros de investigación temáticos ${ }^{5}$.

Línea de investigación, eje temático, monotemático o interdisciplinario en el que confluyen actividades de investigación realizados por uno o más grupos de investigación que tengan resultados visibles en su producción académica y en la formación de recursos humanos mediante el desarrollo de trabajos finales o tesis"6.

Vinculación procede del Latín Vinculatio y hace mención a la acción y efecto de vincular (atar algo en otra cosa, perpetuar algo, someter el comportamiento de alguien al de otra persona, sujetar, asegurar)"7 es decir, tratándose de un determinado estudio 0 actividad: la vinculación significa que debe realizarse con la cooperación de varias disciplinas.

5

http://www.anuies.mx/media/docs/89_2_1_1012161230Reynaga _Obregon_Y_Farfan_Flores Redes Academicas.pdf

$\overline{6}$ http://www.slideshare.net/abelsuing/definicin-de-las-Ineas-deinvestigacin

${ }^{7}$ http://definicion.de/vinculacion/
Investigación: (Del lat. investigatioo, -ōnis). 1. f. Acción y efecto de investigar. Básica.

1. f. investigación que tiene por fin ampliar el conocimiento científico, sin perseguir, en principio, ninguna aplicación práctica"8

También se define como el: "Conjunto de procesos de indagación y búsqueda del conocimiento, orientada al análisis y explicación de las propiedades, estructuras y relaciones de la naturaleza y sus fenómenos, con el objeto de proponer soluciones a su problemática"9.

Miembros: "miembro Del lat. membrum ... 7. m. y f. Individuo que forma parte de un conjunto, comunidad o corporación"10.

"Del latín corpus, el término cuerpo tiene varios usos. Se refiere, por ejemplo, a algo que posee tamaño limitado y que es apreciable por los sentidos, al grupo de sistemas orgánicos que conforman un ente vivo"11

Colaboradores: "Colaboración es la acción y efecto de colaborar. Este verbo refiere a trabajar en conjunto con otra u otras personas para realizar una obra...

Asimismo, en el medio televisivo también ha crecido en los últimos años el uso del término colaborador. Este se emplea para referirse a aquel individuo que participa de manera asidua en un programa, no como presentador sino simplemente aportando sus ideas 0 conocimientos en determinados temas que sean abordados en el mencionado espacio"12.

\footnotetext{
http://buscon.rae.es/drae/srv/search?id=7ok28mpSCDXX2SNekCz

$\frac{\mathrm{s}}{9}$ http://www.uaemex.mx/abogado/docs/Diccionario\%20pdf.pdf

${ }_{10}^{10} \mathrm{http} / / /$ dle.rae.es/?id=PDfN5jE

${ }_{11}$ http://definicion.de/cuerpo/\#ixzz3uu9QR8vr

12 http://definicion.de/colaboracion/\#ixzz3uuADoh2D
} 


\section{METODOLOGIA:}

Planteamiento del Problema: La mayoría de la planta docente con grado de Maestría y Doctorado, contratados con la categoría de asignatura o medio tiempo, por tiempo determinado o definitivo, en todas las Instituciones del Nivel Superior, sean Públicas 0 Privadas, por carecer del perfil deseable reconocido Institucionalmente, únicamente son considerados como colaboradores, no obstante de realizar investigaciones de calidad; características que se consideran una restricción para integrarse como miembros investigadores de los "Cuerpos Académicos (CA), Redes (RI) y Líneas de investigación Científica".

Consecuentemente, la investigación cientíica es limitada, considerando que al modificar los criterios en éste rubro y se permitiera la participación en general en la comunidad de las diversas Instituciones Educativas del Nivel Superior, el resultado sería importante y se reflejaría en el producto, su impacto por su publicación, a nivel Institucional, Nacional e internacionalmente, en beneficio de la sociedad, logrando con ello la solución de sus problemas que tanto los aquejan.

Pregunta de investigación: $\mathrm{Si}$ las Instituciones Educativas del Nivel Superior, modifican sus criterios, relacionados con el perfil deseable, respecto a los miembros investigadores de los Cuerpos Académicos, Redes y Líneas de Investigación Científica, ¿Los Docentes con grado de Maestría o Doctorado, por Asignatura o Medio Tiempo, contratados por tiempo determinado o definitivo, en la Benemérita Universidad Autónoma de Puebla y en general del nivel superior sean Públicas o Privadas, estarían en posibilidad de integrarse como miembros investigadores de los Cuerpos Académicos (CA), Redes (RI) y Líneas de Investigación científica, para de ésta manera integrase a las diversas líneas de investigación científica, enriqueciendo con ello dicha actividad, se lograría realmente incrementar la publicación y difusión de los productos de investigación en beneficio de su Comunidad Institucional, Estudiantil y Social y del ámbito Nacional e Internacional?

Hipótesis: Si los docentes, actualmente guía, coordinador, orientador, facilitador e instructor del proceso aprendizaje-enseñanza, independientemente de su grado de preparación académica, categoría y tipo de contratación, en especial de la Facultad de Contaduría Pública y Administración de la Benemérita Universidad Autónoma de Puebla, y en general de las Instituciones Educativas del Nivel Superior, sean Públicas o Privadas, se constituyen y vinculan como miembros investigadores de los Cuerpos Académicos (CA), Redes y Líneas de Investigación Científica, se generarían resultados importantes en la investigación científica

Justificación: El presente trabajo, tiene como objetivo realizar un análisis del por qué no se incluye a la totalidad de los docentes, actualmente guía, coordinador, orientador, facilitador e instructor del proceso aprendizaje-enseñanza, y sobre todo, de las limitantes que imperan en la integración como miembros investigadores de los "Cuerpos académicos (CA), Redes (RI) y Líneas de Investigación Científica", que por carecer del "perfil deseable", beneficio exclusivo de los docentes con categoría de Tiempo Completo, con grado académico de Doctorado, Maestría y con tipo de contratación como Definitivos.

Y en el caso de los docentes, actualmente guía, coordinador, orientador, facilitador e instructor del proceso aprendizaje-enseñanza, con categoría de Asignatura (hora-clase) y Medio Tiempo, con Grado de Maestría, excepcionalmente con grado de Doctor y con categoría de contratación determinados 0 definitivos, se les acepta en los Cuerpos Académicos como colaboradores en la investigación científica, aun cuando realizan el mismo trabajo que los Miembros de los Cuerpos Académicos, Redes y Líneas de 
Investigación Científica; luego entonces la investigación científica de calidad, es compartida.En éstas condiciones, dicha situación, desmoraliza a los docentes, actualmente guía, coordinador, orientador, facilitador e instructor del proceso aprendizajeenseñanza, en especial, no sólo de la Facultad de Contaduría Pública y Administración, de la Benemérita Universidad Autónoma de Puebla, sino en general a las Instituciones Educativas del Nivel Superior, sean Públicas o Privadas, con grado académico de Maestría o Doctorado, con categoría de Asignatura (hora-clase) y Medio Tiempo, sean determinados o definitivos, lo que además impide su participación como miembros investigadores, tanto en los Cuerpos Académicos, Redes y líneas de investigación científica.

Aunado a lo anterior, las líneas de investigación científica, constituyen otra limitante más, pues no permite que el docente, actualmente guía, coordinador, orientador, facilitador e instructor del proceso aprendizaje-enseñanza, en especial de la Facultad de Contaduría Pública y Administración, de la Benemérita Universidad Autónoma de Puebla y en general a las Instituciones Educativas del Nivel Superior, sean Públicas o Privadas, que demuestran sus competencias, se dediquen a la investigación sobre determinadas líneas de investigación, lo que implicarían resultados en beneficio de los Cuerpos Académicos, Redes y Líneas de Investigación Científica, de la Institución, comunidad estudiantil y social.

Es decir, con los lineamientos sobre la integración de los Cuerpos Académicos, Redes y Líneas de Investigación Científica, se observa que no se permite que haya mayor participación de la totalidad de los docentes, actualmente guía, coordinador, orientador, facilitador e instructor del proceso aprendizajeenseñanza, en especial de la Facultad de Contaduría y Administración de la Benemérita Universidad Autónoma de Puebla y en general de las diversas Instituciones Educativas del Nivel Superior, sean Públicas y Privadas, independientemente de su grado de preparación, categoría y tipo de contratación, como miembros de los Cuerpos Académicos, Redes y Líneas de Investigación Científica.

Considerando además, que si bien es cierto, el derecho inherente a la calidad del ser humano, persona o ciudadano, no era concebido en su origen como sistema de garantías para lograr su desarrollo pleno, se considera con un criterio moderno y universal respecto al reconocimiento de los derechos de todos los seres humanos, por parte del Estado en general, por lo que se considera una violación a sus derechos humanos consagrados en la parte dogmática de nuestra Constitución Política de los Estados Unidos Mexicanos (CPEUM) y de los Tratados Internacionales (TI). Por su parte la primera de las citadas lo considera en su artículo $1^{\circ}$ que establece:

\footnotetext{
En los Estados Unidos Mexicanos todas las personas gozarán de los derechos humanos reconocidos en esta Constitución y en los tratados internacionales de los que el Estado Mexicano sea parte, así como de las garantías para su protección, cuyo ejercicio no podrá restringirse ni suspenderse, salvo en los casos y bajo las condiciones que esta Constitución establece. Las normas relativas a los derechos humanos se interpretarán de conformidad con esta Constitución y con los tratados internacionales de la materia favoreciendo en todo tiempo a las personas la protección más amplia. Todas las autoridades, en el ámbito de sus competencias, tienen la obligación de promover, respetar, proteger y garantizar los derechos humanos de conformidad con los principios de universalidad, interdependencia, indivisibilidad $y$ progresividad. En consecuencia, el Estado deberá prevenir, investigar, sancionar y reparar las violaciones a los derechos humanos, en los términos que establezca la ley. Está prohibida la esclavitud en los Estados Unidos Mexicanos. Los esclavos del extranjero que entren al territorio nacional alcanzarán, por este solo hecho, su libertad y la protección de las leyes. Queda prohibida toda discriminación motivada por origen étnico o nacional, el género, la edad, las discapacidades, la condición social, las condiciones de salud, la religión, las opiniones, las preferencias sexuales, el estado civil o cualquier otra que atente contra la dignidad humana y tenga por objetoanularo menoscabar los derechos y libertades de las personas. ${ }^{13}$
}

\footnotetext{
${ }^{13}$ http://www.diputados.gob.mx/LeyesBiblio/pdf/1_150917.pdf
} 
Mauricio Ortigosa Hernández

Por lo que es indispensable, que se modifiquen los criterios, planteados en el presente trabajo, respecto a la integración de los cuerpos académicos, redes y líneas de investigación científica, con la finalidad de conceder la oportunidad a los docentes, actualmente, permite que haya mayor participación por la totalidad de los docentes, actualmente guía, coordinador, orientador, facilitador e instructor del proceso aprendizaje-enseñanza, en especial de la Facultad de Contaduría y Administración de la Benemérita Universidad Autónoma de Puebla y en general de las diversas Instituciones Educativas del Nivel Superior, sean Públicas y Privadas, independientemente de su grado de preparación académica, de su categoría y tipo de contratación, en la integración como miembros investigadores de los Cuerpos Académicos, Redes y Líneas de Investigación Científica.

Porque, "Todo docente es, por definición un educador, un agente moral que tiene el conocimiento social de contribuir a la formación total de sus alumnos. Ellos tienen el deben de influir en la formación integral de sus alumnos, pero no imponiéndoles, sino creando las condiciones, logrando el clima adecuado y orientando las tareas al estímulo para la construcción de valores propios"14.

Indiscutible es e innegable, que la investigación científica, está fuertemente relacionada con el desarrollo social y económico de un país determinado, quienes además se distinguen por sus diversos sistemas de investigación científica, dependiendo de sus estrategias y políticas más 0 menos adecuadas dentro del campo tecnológico y científico, lo que influye para la constitución y consolidación de centros de investigación e instituciones académicas, que requieren de importante inversión financiera, para la formación de sus recursos humanos, cuyo objetivo lo constituye la infraestructura necesaria, que darán resultados a

\footnotetext{
${ }^{14}$ García Flores, Jacinto. Compilador. Desarrollo Institucional y Educación Continua. edición 2009. Edit. Benemérita Universidad Autónoma de Puebla, Vicerrectoría de Docencia, Dirección de Fomento Editorial. p. 21
}

mediano o largo plazo, por lo que se considera que nuestra Institución Benemérita Universidad Autónoma de Puebla, debe invertir recursos económicos, en la preparación de sus docentes, independientemente de su grado de preparación académica, categoría y tipo de contratación, con la finalidad de que se dediquen a la investigación científica.

Lo anterior, permitirá que la investigación científica, no sólo en nuestra Alma Máter, sino en general de las Instituciones Educativas del Nivel Superior, sean Públicas o Privadas, en la que deben estar inmersos la generalidad de los docentes, actualmente guía, coordinador, orientador, facilitador e instructor del proceso aprendizaje-enseñanza, independientemente de su grado de preparación académica, categoría y tipo de contratación, con la finalidad de alcanzar en el ámbito de la investigación científica su máximo índice de desarrollo, no sólo a nivel regional, local, nacional, sino incluso a nivel internacional, lo anterior, permitirá fortalecer a nuestra Alma Máter, en beneficio de nuestro país México, que se distinguirá de los demás países, incluso con un nivel de desarrollo económico elevado, no sólo a nivel latinoamericano, sino a nivel mundial, en el que es innegable que en aquella, la investigación no se impulsó de la misma manera que en ésta, en el que no puede haber comparación, toda vez que es importante en general su contexto, que en determinado momento influye para su deterioro o impulso, en el presente, relativo a su investigación científica.

Mismo contexto que es determinante, incluso para evitar en la medida de lo posible, la migración de la clase científica, con la consecuente disminución obviamente la actividad científica en nuestro país, difícil de recuperar, como se desprende de los datos duros proporcionados por el Instituto Nacional de Estadística, Geografía e Informática (INEGI). Nuestro país, se ve favorecido por los años del cincuenta al setenta, por la creación en materia educativa de sus centros de investigación, que en muchos de los casos fueron frenados por los efectos de la deuda externa, 
que incluso se vio limitado para el financiamiento de sus proyectos de investigación sobre todo científica.

Posteriormente, se considera la creciente necesidad de una sociedad educada, con la finalidad de en un futuro integrarse a una sociedad competitiva $y$ globalizada, logrando la igualdad mundial, como sucede actualmente y de ésta manera, erradicar la desigualdad, que están padeciendo los docentes, independientemente de su grado de preparación académica, categoría y tipo de contratación, referidos, por limitar su derecho en los términos indicados con anterioridad.

Por lo que México, en su afán de desarrollo en la investigación científica, que impulsó en su momento, el aparato gubernamental a través de la Secretaría de Educación Pública y diversas dependencias como las instituciones de educación superior públicas, incluyendo los esfuerzos privados, muchas veces apoyada por las instituciones de carácter internacional, como la UNESCO, la Organización de Estados Americanos. (OEA), que se concreta en varios rubros, destacando por su importancia, la investigación que realizan las universidades.

Lo anterior implica, una serie de restricciones en cuanto a recursos económicos, pero que los centros de investigación que lograron sobrevivir, notaron un cambio en cuando al apoyo económico que se les brindó en forma oportuna.

Es importante, además, descifrar y manejar, el léxico empleado en la investigación científica, que servirá como punto de partida para realizar nuevas investigaciones. Para incursionar en éste contexto, la Vicerrectoría de Docencia, de la Benemérita Universidad Autónoma de Puebla, a través de la Escuela de Formación Docente, ofertó el Diplomado sobre Investigación Científica, en su Tercer y Cuarto módulo, impartido por el profesor investigador Dr. Alejandro Farfán Balanzategui ${ }^{15}$, hizo la siguiente recomendación:

\footnotetext{
${ }^{15}$ Farfán Balanzategui, Alejandro. Dr. y Profesor Investigador. Diplomado en Metodología de la Investigación. Escuela de
}

\begin{abstract}
...como dinámica: para ser un buen investigador científico, es indispensable, que por sesión, se realice la investigación del significado de dos o tres términos, de preferencia los empleados en la investigación científica, para familiarizarse con el significado de los esquemas del diccionario... para en su oportunidad realizar un trabajo de investigación científica de calidad, en el que según su concepción lo denominó conocimiento científico, con su correspondiente fiabilidad y valía de aprendizaje por conceptos, ...
\end{abstract}

Ahora bien, "para asumir la tarea de investigar se hace necesario el desarrollo de procesos de pensamiento, de habilidades y actitudes que conduzcan al ejercicio para la investigación. Se requiere de una serie de transformaciones que nos lleven de sujetos constituidos para determinadas prácticas profesionales, a la formación de sujetos epistémicos (Testa Mario, 1999), es decir formar sujetos que puedan apropiarse del denominado conocimiento avanzado, el cual se caracteriza por (Clark, Burton, 1993):
a) Ser especializado, circunscrito a una materia de estudio, la que continuamente se subdivide para formar otros campos y especializaciones.
b) Abierto a tener un compromiso con lo nuevo, con 10 desconocido, con lo incierto, con lo cambiante, en una actitud permanente de búsqueda.
c) Autónomo porque cobra independencia del conocimiento general del que originalmente participó.
c) Portador de herencias culturales.

La formación para la investigación es entendida como un proceso que implica prácticas y actores diversos (en el presente trabajo constituido por docentes y estudiantes), en el que la intervención de los formadores como mediadores humanos, se concreta en un quehacer académico y que consiste en promover y facilitar, preferentemente de manera sistematizada (no necesariamente escolarizada), el

Formación Docente, Benemérita Universidad Autónoma de Puebla, con fecha cinco de agosto del año dos mil quince. 
Mauricio Ortigosa Hernández

acceso a los conocimientos, el desarrollo de las habilidades, hábitos y actitudes, y la interiorización de valores, que demanda la realización de la práctica llamada investigación (Moreno Guadalupe, 2005).

\section{RESULTADOS:}

Nuestro sistema educativo inmerso en el nuevo orden globalizado, es una realidad que busca la competitividad, eficacia, eficiencia y todo género de calidad, a la que nos enfrentamos todos los seres humanos del globo terráqueo sin excepción, fenómeno que trasciende a todo tipo de Instituciones tanto Públicas como Privadas, incluyendo a las Organizaciones y a la Sociedad en General, no quedan exentas de ello las Instituciones Educativas, en sus diferentes modalidades y niveles, incluyendo las del Nivel Superior, en este sentido el capital humano es un factor trascendente.

Las Instituciones de Educación Superior, específicamente la facultad de Contaduría Pública y Administración de la Benemérita Universidad Autónoma de Puebla y en general las de Carácter Público o Privado, considerando sistemáticamente las tendencias que se dan en el mundo globalizado, obviamente el laboral, en los sectores científicos, tecnológicos, políticos y económicos, relacionados con su contribución en la Educación Superior, se identifican por el grado de mejora que tienen en la formación de su personal docente, actualmente guías, coordinadores, orientadores, facilitadores e instructores del proceso aprendizaje-enseñanza, de los estudiantes, en los planes y programas de estudio y la investigación científica, para fortalecer su desarrollo integral, que incide en la construcción de aprendizajes significativos de los estudiantes, futuros profesionistas, porque la contribución de la educación a la sociedad está definida por el grado de mejora que se tenga en la formación del personal docente, los planes de estudio y la investigación científica. Conforme a la Gaceta Universidad ${ }^{16}$, Órgano Oficial de la Benemérita Universidad Autónoma de Puebla, reconoce que, son tres niveles de Cuerpos Académicos:

\footnotetext{
a) Consolidados: Un cuerpo Académico se considera Consolidado, cuando la mayoría de sus miembros tiene alto nivel académico que los capacita para generar y aplicar el conocimiento, así como para la formación de recursos humanos.

Características: Tienen una intensa actividad académica, que se refleja, además, en los Congresos, Seminarios, Talleres de manera regular y frecuente y mantienen una intensa participación en redes de intercambio académico y con organismos e instituciones nacionales y extranjeras.

b) Se considera que un Cuerpo Académico está en Consolidación cuando más de la mitad de sus integrantes tiene alta calidad en sus tareas de investigación y docencia, sobre todo en la generación y aplicación innovadora del conocimiento; por lo menos la tercera parte de quienes lo integran cuenta con amplia experiencia en docencia.

c) Un Cuerpo Académico en formación significa que al menos la mitad de sus integrantes tiene el reconocimiento del perfil deseable, tienen definidas sus líneas de investigación e identificados algunos cuerpos académicos afines y de alto nivel, de otras instituciones nacionales 0 extranjeras con las cuales desean establecer contactos.
}

Al respecto, la Gaceta Universidad BUAP, confirma lo anterior, en los siguientes términos: "Calidad y prestigio académico. BUAP, primer lugar nacional en Cuerpos Académicos consolidados: La alta producción científica de la Benemérita Universidad Autónoma de Puebla y el número de citas que genera están relacionados con su planta académica. Por tercer año consecutivo, en 2015 se posicionó como la universidad pública estatal con más Cuerpos Académicos Consolidados del país, reconocidos por la Secretaría de Educación Pública.

16 Gaceta Universidad. Órgano Oficial de la Benemérita Universidad Autónoma de Puebla. No. 173. Noviembre 2013. Puebla, 2013. Presentación. 
La Máxima Casa de Estudios de Puebla, tiene en total 186 Cuerpos Académicos: 82 son consolidados, 76 están en consolidación y 28 en formación, pilares de la calidad educativa de la BUAP, ya que son grupos de profesores de tiempo completo que comparten una o varias líneas de generación y aplicación del conocimiento, y en cuya labor han sido respaldados por el Programa Institucional de Apoyo a Cuerpos Académicos, impulsado por el Rector Alfonso Esparza Ortiz.

En ese indicador, en el que se consideran criterios de calidad académica, como docencia, investigación, publicaciones y formación de redes de investigación con grupos nacionales e internacionales, entre otros, le sigue la Universidad Autónoma de Nuevo León con 74 Cuerpos Académicos Consolidados y la Universidad de Guadalajara con 73; mientras que el resto de las universidades tienen menos de 40.

Los rasgos de los Cuerpos Académicos son:
Conforme a lo planteado con anterioridad, se pretende que exclusivamente los docentes de Tiempo Completo en especial de las Facultades de Contaduría Pública y Administración, de la Benemérita Universidad Autónoma de Puebla, así como de las Instituciones Educativas del Nivel Superior, sean Públicas o Privadas, con su integración a los Cuerpos Académicos, Redes y Líneas de Investigación Científica, por tener el perfil deseable y contribuyen por su alto nivel académico, son capacitados para generar y aplicar el conocimiento, pero realmente qué sucede con los docentes de las citadas Instituciones Educativas, con grado académico, categoría y tipo de contratación, que también forman parte del personal docente no sólo de las facultades citadas, sino en general de las Instituciones de Educación Superior que no cuentan con dicha distinción, por circunstancias adversas 0 simplemente un docente de hora clase-determinado, que no cuenta con las condiciones necesarias para acceder a obtener la categoría y perfil deseable, por lo que, simplemente no están en condiciones de continuar con su preparación y con ello acceder a los Cuerpos Académicos (CA) consecuentemente Redes (RI) y Líneas de Investigación científica; acaso no contribuyen en la generación y aplicación del conocimiento.

En virtud del anterior razonamiento, se considera que dichos docentes, que no reúnen el perfil deseable, también tienen la capacidad de ser miembros, no colaboradores de Cuerpos Académicos (CA) consecuentemente Redes (RI) y Líneas de Investigación científica, para contribuir en gran medida a aportar sus conocimientos adquiridos en beneficio no sólo del futuro Contador Público y Administrador en sus diversas modalidades, que constituyen una verdadera herramienta que les permiten desarrollarse en el ambiente laboral y estos conocimientos corresponden a las necesidades de las Instituciones Educativas, tanto Públicas como Privadas, Organizaciones y Sociedad en General, quienes requieren de sus egresados, la capacidad y 
Mauricio Ortigosa Hernández

competencia, para desempeñarse efectivamente como profesionistas, en los diversos puestos de trabajo disponibles, requiriendo se integren al campo laboral por cumplir con las competencias y conocimientos necesarios, pero sobre todo porque adquirieron las habilidades, destrezas, aptitudes, actitudes y valores, capaces de aplicar sus conocimientos en la resolución de sus respectivos problemas.

Los docentes que no tienen la categoría de Tiempo Completo, algunos con Grado de Maestría 0 Doctorado, contratados por Asignatura, Medio Tiempo, sean determinados o definitivos en sus diferentes modalidades incluso perciben un salario inferior a los docentes de carrera (que gozan de infinidad de prebendas), luego entonces, los citados docentes, que no cuentan con el grado de Maestría o Doctorado, carecen de la capacidad económica para continuar su preparación de Maestría, Doctorado, Pos doctorado, etc., por no ser autofinanciables, no pueden constituirse como miembros, de los citados grupos académicos, solamente como colaboradores, en los Cuerpos Académicos, Redes y Líneas de Investigación Científica, olvidando que son parte integrante de las Instituciones Educativas del Nivel Superior y, por lo tanto, éstas, son las principales proveedoras de profesionistas que se incorporan al mundo de los negocios, en sus diferentes modalidades, ya sea como emprendedores o como capital humano en los diferentes ámbitos, por ser altamente calificados y competentes, como consecuencia de sus planes y programas de estudio, tendencias requeridas en el nivel internacional, nacional, regional y local, proyectando conocimientos significativos, pertinentes, de calidad y con óptimos resultados.

\section{Discusión:}

Crítica: Para efectos del presente trabajo de investigación científica, es necesario hacer la siguiente crítica: Los docentes, actualmente guía, coordinador, orientador, facilitador e instructor del proceso aprendizaje-enseñanza, en especial de la Facultad de Contaduría y Administración de la Benemérita Universidad Autónoma de Puebla y en general de las diversas Instituciones Educativas del Nivel Superior, sean Públicas y Privadas, independientemente de su grado de preparación académica, categoría y tipo de contratación, que no reúnen el perfil deseable, son limitados para integrarse como miembros de los Cuerpos Académicos (CA), Redes (RI) y Líneas de Investigación científica, aun cuando se les reconoce como colaboradores, lo que se considera demerita su trabajo de investigación, porque contribuyen no sólo de manera asidua simplemente aportando sus ideas 0 conocimientos en determinados temas, sino que hacen una verdadera aportación de sus conocimientos científicos, en los proyectos de investigación científica.

Motivo por el cual, es innegable que, aun cuando específicamente, los docentes referidos, poseen la preparación necesaria sobre la investigación científica, por demostrar sus competencias en distintas áreas y capacidad para integrarse como miembros a los Cuerpos Académicos, Redes y Líneas de Investigación Científica, al no permitir su ingreso, se les limita en su derecho a participar en los citados grupos académicos e incluso en la investigación científica.

Los docentes, actualmente guía, coordinador, orientador, facilitador e instructor del proceso aprendizaje-enseñanza, en especial de la Facultad de Contaduría y Administración de la Benemérita Universidad Autónoma de Puebla y en general de las diversas Instituciones Educativas del Nivel Superior, 
sean Públicas y Privadas, independientemente de su grado de preparación académica, categoría y tipo de contratación, que no reúnen el perfil deseable, si no existiese una limitante para integrarse como miembros de los Cuerpos Académicos (CA), Redes (RI) y Líneas de Investigación científica y que conforme a los diferentes lineamientos Institucionales establecidos, si no existiese dicha restricción para formar parte de los citados grupos citados, se aprovecharía la oportunidad y eficiencia del recurso humano que tienen a su servicio en este rubro.

El Reglamento de Cuerpos Académicos de la Universidad Tecnológica de Puebla ${ }^{17}$, que a continuación se presenta, tiene mayor apertura para la integración de los mismos y destaca la diversidad de las líneas de investigación y del trabajo académico interdisciplinario a diferencia de los lineamientos establecidos por la Benemérita Universidad Autónoma de Puebla (BUAP).

\begin{abstract}
Capítulo 1 Disposiciones generales y definiciones:
Artículo 1. La integración y funcionamiento de los Cuerpos Académicos de la Universidad.

Tecnológica de Puebla, así como la permanencia de sus integrantes estarán sujetos a los Instrumentos Jurídicos y la normatividad aplicable en la UTP y en específico, a lo estipulado en el presente Reglamento.
\end{abstract}

Artículo 2. Para efectos de este Reglamento, se entenderá por Cuerpo Académico, al grupo que, como mínimo, está constituido por 3 profesores de tiempo completo, con designación de titular y que comparten una o varias líneas de investigación o de estudio, de temas disciplinares o multidisciplinares, y un conjunto de objetivos y metas académicos.

Artículo 3. Los cuerpos académicos (CA's) en la Universidad Tecnológica de Puebla (UTP) se integran con miembros titulares y asociados, que comparten un conjunto de metas académicas con los siguientes objetivos:

a) Definen y cultivan una o varias líneas de investigación aplicada y desarrollo tecnológico (LIADT), las cuales se orientan principalmente a la aplicación innovadora del

17

http://www2.utpuebla.edu.mx/l/Reglamento Cuerpos Academic os.pdf. conocimiento.b) Forman recursos humanos de alto nivel en las áreas de su competencia.c) Fomentan la mejora continua de la calidad de los programas educativos en los que participan.

I. Se entiende por miembro titular, al Profesor universitario de tiempo completo, con nombramiento que implique una dedicación mínima de 40 horas/semana en la institución. Con nivel de habilitación mínimo de licenciatura ó preferentemente de maestría ó especialidad tecnológica, que cuente con nombramiento de titularidad emitido por Secretaría Académica, y realice las siguientes funciones:

a) Docencia, b) Líneas de investigación aplicada y desarrollo, c) Gestión académica y de vinculación y d) Tutorías. II. Se entiende por miembros asociados, a los alumnos y profesores que colaboren parcialmente en la generación de productos del CA, y sean designados por los miembros titulares del $C A$.

Porque según Lewin ${ }^{18}$ y sus seguidores, citado por la Dra. Blanca Heredia Rubio, manifiesta: "el proceso de investigación está al servicio del cambio social". A la Benemérita Universidad Autónoma de Puebla, se le ha signado Institución Educativa del Nivel Superior como "BUAP, LIDER EN INVESTIGACIÓN", denominación que ha adquirido por el interés del $\mathrm{C}$. Rector M. A. Alfonso Esparza Ortiz, como se desprende de la Gaceta Universitaria número $173^{19}$, en los siguientes términos:

La investigación es una de las funciones sustantivas que
realizan las universidades, en ella se forman científicos
que coadyuvan a resolver los temas más importantes del
país y del mundo entero, en éste sentido la Benemérita
Universidad Autónoma de Puebla es hoy una de las
instituciones de educación superior reconocida, nacional e
internacionalmente, por la calidad de sus investigaciones y
la relevancia de algunas de sus líneas de investigación.

Las autoridades universitarias han puesto énfasis en la producción científica y en la calidad de la misma, sus investigadores cuentan con estímulos y una infraestructura científica moderna lo que ha permitido que su trabajo sea publicado en revistas, además de colaborar en trabajos internacionales...

\footnotetext{
${ }^{18}$ Op. Cit

19 Gaceta Universidad. Órgano Oficial de la Benemérita Universidad Autónoma de Puebla. No. 173. Noviembre 2013.

Puebla, 2013. presentación
} 
Mauricio Ortigosa Hernández

El liderazgo de la academia y la investigación en la Buap se encuentra cimentado en la calidad de su planta académica ya que, de 1736 profesores de tiempo completo, el 90 por ciento cuenta con estudios de posgrado y el 55 por ciento tiene el reconocimiento de perfil deseable Promep (actualmente Prodep).

Del Padrón Institucional de Investigadores 469 pertenecen al Sistema Nacional de Investigadores, asi mismo, 44 de un total de 83 maestrías y doctorados se encuentran hoy en el Padrón Nacional de Posgrados de Calidad, 2 de ellos en el nivel de competencia internacional, además de ser la Universidad Pública con el mayor número de Cuerpos Académicos Consolidados y en Consolidación, grupos de investigación donde participan más del 40 por ciento de los profesores de tiempo completo.

Lo anterior es el resultado del compromiso de las autoridades de la Buap que hoy permiten que la Máxima casa de estudios del Estado sea considerada como una de las mejores Instituciones de Educación Superior del País y de América Latina".

De lo anterior se desprende, que las autoridades de Nuestra Alma Máter, apoyado por las Instituciones Educativas del Nivel Superior, tienen especial interés en posicionarla en la actividad científica, como una de las mejores sobre todo a Nivel Latinoamérica, que con la integración de la totalidad de los docentes, independientemente de su grado académico, categoría, tipo de contratación, como miembros investigadores de los diversos Cuerpos Académicos (CA), Redes (RI) y Líneas de Investigación Científica, incluyendo a sus estudiantes, se logrará mayor investigación científica, misma que al lograr su difusión, estamos seguros, que de ésta manera se logrará posicionar a nivel no sólo de Latinoamérica, sino a Nivel Mundial.

Es importante destacar, que actualmente, la Benemérita Universidad Autónoma de Puebla, a través de su Rector, M. A. Alfonso Esparza Ortiz, ha mostrado desde siempre, su interés para lograr la vinculación, impulsar y difundir la investigación científica, como se desprende de la segunda parte de la entrevista con el Secretario General Ejecutivo de la Asociación Nacional de Universidades e Instituciones de Educación Superior (ANUIES) ${ }^{20}$, Mtro. Jaime Valls Esponda, realizada por el Dr. Jorge Pérez Mejía, en los siguientes términos:

\begin{abstract}
Dr. Jorge: A mí me llama mucho la atención que el Slogan de Responsabilidad Social Universitaria "Retos Globales y Locales", que anuncia la asamblea de la ANUIES en el sentido que pareciese que antes se hablaba de temas tradicionales clásicos y hoy entra a la mesa en éstos 5 puntos que se señalan Responsabilidad Social Universitaria incluso hoy se está trabajando en talleres, en mesas, tenemos un taller...podemos pensar en que las Universidades generen profesionistas sí, pero ante todo buenos ciudadanos, eso es fundamental y hoy se lleva a la mesa con unos puntos centrales en que la Anuies está trabajando. Dr. Jaime... Es construir ciudadanía de manera conjunta y mayor vinculación, las Universidades no pueden estar ajenas a su entorno, a su realidad y menos las Universidades Públicas que se financian de recursos fiscales, de recursos públicos que los mexicanos pagan sus impuestos, entonces tiene que haber ésta vinculación, ésta responsabilidad social y ver como si hay beneficios concretos en los temas de investigación...
\end{abstract}

En estas condiciones y conforme al estudio realizado en el presente trabajo de investigación científica, es necesario destacar, la importancia de que los docentes, actualmente guía, coordinador, orientador, facilitador e instructor del proceso aprendizajeenseñanza, en especial de la Facultad de Contaduría y Administración de la Benemérita Universidad Autónoma de Puebla y en general de las diversas Instituciones Educativas del Nivel Superior, sean Públicas y Privadas, independientemente de su grado de preparación académica, categoría y tipo de contratación, que, aun cuando no reúnen el perfil deseable, no deben estar limitados para integrarse como miembros investigadores de los Cuerpos Académicos (CA), Redes (RI) y Líneas de Investigación Científica.

Es indispensable, dar cumplimiento a los Lineamientos establecidos como metodología de investigación científica, con la finalidad de no lamentar 0 errar en dicha actividad, que sería en perjuicio tanto de la Institución, docentes, estudiantes

\footnotetext{
20 Pérez Mejía, Jorge. Educación en movimiento. La Opinión Universitaria. Benemérita Universidad Autónoma de Puebla. 7 de diciembre del 2015. p 9.
} 
y de la sociedad en general, lo que nos depararía un perjuicio, no sólo como Institución Pública, Educativa del Nivel Superior incluyendo el bagaje de investigadores, comunidad estudiantil, sociedad, además de los ámbitos Regional, Local, Estatal, Nacional e internacionalmente, y así, Jaime Torres Bodet $^{21}$, se expresa en los siguientes términos:

\begin{abstract}
Cuando vuestros profesores os señalaron en las escuelas como un éxito en la civilización-los inventos más ingeniosos de la mecánica y de la química, las elaboraciones más complicadas del arte de razonar y de persuadir- ha perdido súbitamente prestigio de afirmación, y en vez de exaltar la vida, pretende exaltar el odio, el mal, la muerte, lo negativo.
\end{abstract}

Os habiais educado en el fervor de la inteligencia. Y la inteligencia se ha convertido en una trágica antagonista. Os habiais alistado para rendir tributo a la ciencia humana. Y la ciencia humana se ha transformado en una máquina de agresión. El aeroplano y las radiocomunicaciones hoy parecian conquistas de paz para llegar más de prisa a vuestros hermanos. Ved lo que llevan ahora los aviones: bombas cargadas con explosivos de efectos cada vez más desastrosos y arrasadores. $Y$ recordad to que las dictaduras han transmitido desde las difusoras totalitarias: cólera y amenazas, traición y embuste, ira y crueldad.

¿Qué significa ese espectáculo doloroso? ¿Una defección de las verdades espirituales?

¿Un cambio imprevisto de signo en el balance de todo nuestro progreso?... De ningún modo. Ni la filosofía, ni la ciencia, ni el arte, tienen la culpa del absurdo que de ellos han hecho quienes desencadenaron esta inmensa conflagración en la cual, para orgullo de México, hemos aceptado con entereza nuestro papel".

Propuesta: Al respecto, que las Instituciones Educativas Federales, del Nivel Superior (SEP) deben modificar los lineamientos para la conformación de los Cuerpos Académicos (CA), Redes (RI) y Líneas de Investigación Científica. Así mismo, la Benemérita Universidad Autónoma de Puebla, debe reestructurar sus políticas educativas para:

1. Modificar sus políticas de integración de los Cuerpos Académicos (CA), Redes (RI) y Líneas de

\footnotetext{
21 Torres Bodet, Jaime 1902-1974. Pensamiento Educativo de Jaime Torres Bodet (1943-1964)/ Compilador: Alberto Enríquez Perea. México. ed. 2012, Edit. El Colegio Nacional, 2012. P 72 ISBN: 978-607-724-031-0
}

Investigación Científica, que permita la incorporación de sus docentes actualmente guía, coordinador, orientador, facilitador e instructor del proceso aprendizaje-enseñanza, en especial de la Facultad de Contaduría y Administración de la Benemérita Universidad Autónoma de Puebla y en general de las diversas Instituciones Educativas del Nivel Superior, sean Públicas y Privadas, independientemente de que reúnan o no el perfil deseable e integrarse como miembros investigadores de los mismos, previa demostración de sus competencias.

2. Fomentar y Promover, la vinculación de sus docentes, independientemente de su grado de preparación académica, categoría y tipo de contratación, que sin reunir el perfil deseable, para que se constituyan como miembros investigadores de los Cuerpos Académicos (CA), Redes (RI)y Líneas de Investigación Científica.

3. Promover, la vinculación de sus docentes, con la finalidad de avanzar en el rubro de la investigación científica, obteniendo mayor producto y su difusión, con su correspondiente beneficio Regional, Local, Nacional e Internacionalmente, Institucional, Comunidad Estudiantil y Sociedad en General.

\section{CONCLUSIONES:}

1. La Secretaría de Educación Pública Federal, debe modificar sus lineamientos, respecto a la conformación como miembros de los Cuerpos Académicos (CA), Redes (RI) y Líneas de Investigación Científica, respecto a los docentes, actualmente guía, coordinador, orientador, facilitador e instructor del proceso aprendizaje-enseñanza, en especial de la Facultad de Contaduría y Administración de la Benemérita Universidad Autónoma de Puebla y en general de las diversas Instituciones Educativas del Nivel Superior, sean Públicas y Privadas, independientemente de que reúnan o no el perfil deseable. 
Mauricio Ortigosa Hernández

2. Así mismo, la Secretaría de Educación Pública Federal, debe modificar sus lineamientos, respecto a la conformación como miembros de los Cuerpos Académicos (CA), Redes (RI) y Líneas de Investigación Científica, respecto a los docentes, de la Facultad de Contaduría y Administración de la Benemérita Universidad Autónoma de Puebla y en general de las diversas Instituciones Educativas del Nivel Superior, sean Públicas y Privadas, independientemente de su grado de preparación académica, categoría y tipo de contratación.

3. La Benemérita Universidad Autónoma de Puebla, debe reestructurar sus políticas educativas de integración de los Cuerpos Académicos (CA), Redes (RI) y Líneas de Investigación Científica, que permita la incorporación de sus docentes, independientemente de que reúnan o no el perfil deseable e integrarse como miembros investigadores de los mismos, previa demostración de sus competencias.

4. Fomentar, la vinculación de sus docentes, que sin reunir el perfil deseable, se constituyan como miembros investigadores de los Cuerpos Académicos (CA), Redes (RI)y Líneas de Investigación Científica.

5. Promover, la vinculación de sus docentes para que se constituyan como miembros investigadores de los Cuerpos Académicos (CA), Redes (RI)y Líneas de Investigación Científica, independientemente de su grado de preparación académica, categoría y tipo de contratación.

6. La Benemérita Universidad Autónoma de Puebla, debe promover, la vinculación de sus docentes, para que se constituyan como miembros investigadores de los Cuerpos Académicos (CA), Redes (RI) y Líneas de Investigación Científica y avanzar en el rubro de la investigación científica, obteniendo mayor producto y su difusión, con su correspondiente beneficio Regional, Local, Nacional e Internacionalmente, Institucional, Comunidad Estudiantil y Sociedad en General.

\section{REFERENCIAS:}

Benemérita Universidad Autónoma de Puebla. Modelo Universitario Minerva. Fundamentos Modelo Universitario Minerva, pp. 69 a 72.

Farfán Balanzategui, Alejandro. Dr. y Profesor Investigador. Diplomado en Metodología de la Investigación. Escuela de Formación Docente, Benemérita Universidad Autónoma de Puebla, 2015.

Gaceta Universidad. Órgano Oficial de la Benemérita Universidad Autónoma de Puebla. No. 132. Nueva Época. Marzo 2010. Puebla, 2010. p. 16.

Gaceta Universidad. Órgano Oficial de la Benemérita Universidad Autónoma de Puebla. No. 173. Noviembre 2013. Puebla, 2013. Presentación.

Gaceta Universidad BUAP. Órgano oficial de la Benemérita Universidad Autónoma de Puebla. Año XXXIV/No. 196/ Octubre 2015/2do. Informe de Labores Rector Alfonso Esparza, pp. 19 y 20.

García Flores, Jacinto. Compilador. Desarrollo Institucional y Educación Continua. Edición 2009. Edit. Benemérita Universidad Autónoma de Puebla, Vicerrectoría de Docencia, Dirección de Fomento Editorial. p. 21

Heredia Rubio, Blanca. Innovación en las escuelas. Primera ed. Edit. Colección: Antologías didácticas INITE-OCDE. México, 2008. p 227 y 228.

Pérez Mejía, Jorge. Educación en movimiento. La Opinión Universitaria. Benemérita 
ISSN: 1794-9920 Impreso / Electrónico 2500-9338

Agosto - Diciembre de 2019 - Volumen 19 Número 2, Año 2019 Págs. 24 - 40

Universidad Autónoma de Puebla. 7 de diciembre del 2015. p 9

\section{FUENTES ELECTRÓNICAS}

http://www.uasnet.mx/caeco/, Encuentro Nacional "Cuerpos académicos y redes de colaboración. Experiencias y perspectivas". Los días 31 de enero, 1 y 2 de febrero de 2008.

$\underline{\text { www.uthh.edu.mx/file_manager/doc }}$

http://www.slideshare.net/abelsuing/definicin-de-las-Ineasde-investigacin

http://definicion.de/vinculacion/

http://www2.utpuebla.edu.mx///Reglamento_Cuerpos_Acad emicos.pdf.

www.diputados.gob.mx/LeyesBiblio/pdf

http://www.anuies.mx/media/docs/89_2_1_1012161230Rey

naga_Obregon_y_Farfan_Flores_Redes_Academicas.pdf

http://buscon.rae.es/drae/srv/search?id=7ok28mpSCDXX2

SNekCzs

http://www.uaemex.mx/abogado/docs/Diccionario\%20pdf.p

df

http://dle.rae.es/?id=PDfN5jE

http://definicion.de/cuerpo/\#ixzz3uu9QR8vr

http://definicion.de/colaboracion/\#ixzz3uuADoh2D 